\title{
Improved Treatment Effect of Triamcinolone Acetonide Extended-Release in Patients with Concordant Baseline Pain Scores on the Average Daily Pain and Western Ontario and McMaster Universities Osteoarthritis Index Pain Scales
}

\author{
Edgar Ross · Nathaniel P. Katz • Philip G. Conaghan • Alan Kivitz • Dennis C. Turk • \\ Andrew I. Spitzer · Deryk G. Jones · Ryan K. Lanier · Amy Cinar · Joelle Lufkin · Scott D. Kelley
}

Received: August 14, 2021 / Accepted: October 20, 2021 / Published online: November 17, 2021

(C) The Author(s) 2021

\section{ABSTRACT}

Introduction: A phase 3 randomized controlled study comparing triamcinolone acetonide extended-release (TA-ER) to conventional TA crystalline suspension (TAcs) reported variable efficacy results. Enrollment criteria may have contributed to this discrepancy, as moderate-tosevere average daily pain (ADP) was required at baseline, whereas no limitations were placed on Western Ontario and McMaster Universities Osteoarthritis Index (WOMAC-A) pain severity.

\section{E. Ross $(\varangle)$}

Brigham and Women's Hospital and Harvard

Medical School, 75 Francis Street, Boston, MA 02115, USA

e-mail: elross@bwh.harvard.edu

\section{N. P. Katz · R. K. Lanier}

Analgesic Solutions, 321 Commonwealth Rd,

Wayland, MA 01778, USA

P. G. Conaghan

Leeds Institute of Rheumatic and Musculoskeletal

Medicine, University of Leeds and NIHR Leeds

Biomedical Research Centre, Leeds LS7 4SA, UK

\section{A. Kivitz}

Altoona Center for Clinical Research,

178 Meadowbrook Lane, P.O. Box 1018,

Duncansville, PA 16635, USA

D. C. Turk

Washington Medicine, Box 356540, 1949 NE Pacific

Street, Seattle, WA 98195, USA
We conducted a post hoc sensitivity analysis to compare treatment effects in patients reporting moderate-to-severe osteoarthritis (OA) pain on both scales.

Methods: Participants $>40$ years old with symptomatic knee OA were randomly assigned to a single intra-articular injection of TA-ER $32 \mathrm{mg}$, TAcs $40 \mathrm{mg}$, or saline-placebo and followed for 24 weeks. Patient-reported ADP, WOMAC-A, rescue medication usage, and adverse events (AEs) were assessed. Participants who reported moderate-to-severe $\mathrm{OA}$ pain at baseline using both instruments (ADP $\geq 5$ to $\leq 9$, maximum 10 and WOMAC-A $\geq 2$,

\section{A. I. Spitzer \\ Department of Orthopaedic Surgery, Cedars-Sinai Medical Center, 444 S. San Vicente Blvd \#603, Los Angeles, CA 90048, USA}

D. G. Jones

Ochsner Sports Medicine Institute, $1221 \mathrm{~S}$.

Clearview Pkwy, Harahan, LA 70121, USA

\section{A. Cinar · J. Lufkin · S. D. Kelley}

Flexion Therapeutics Inc., 10 Mall Road, Suite 301, Burlington, MA 01803, USA 
maximum 4) were categorized as "concordant" pain reporters; patients with baseline moderateto-severe OA on ADP only were termed "discordant" pain reporters.

Results: Two-hundred-ninety-two concordant pain reporters of 484 total subjects received TA-ER $32 \mathrm{mg}(n=95)$, TAcs $40 \mathrm{mg}(n=100)$, or saline-placebo $(n=97)$. Baseline characteristics and $\mathrm{AE}$ profiles of the concordant and discordant pain responders were consistent with the full analysis population. Among concordant pain reporters, TA-ER significantly $(p<0.05)$ improved ADP scores vs. TAcs (weeks 5-19; areaunder-the-effect $\left.[\mathrm{AUE}]_{\text {weeks1-12; }} \mathrm{AUE}_{\text {weeks1-24 }}\right)$ and saline-placebo (weeks 1-20; AUE weeks1-12; $\left.\mathrm{AUE}_{\text {weeks1-24 }}\right)$. At week 12, a higher proportion reported no knee pain $(\mathrm{ADP}=0)$ with $\mathrm{TA}-\mathrm{ER}$ $(\sim 28 \%)$ vs. TAcs $(\sim 8 \%)$. TA-ER significantly improved WOMAC-A vs. TAcs at weeks 4,8 , and 12 , with significant reduction in rescue medication usage observed with TA-ER from weeks 2 to 20 vs. TAcs.

Conclusions: In patients reporting moderateto-severe knee OA pain at baseline based on concordant ADP and WOMAC-A scores, TA-ER provided statistically significant pain relief for $\geq 12$ weeks compared with conventional TAcs.

Trial Registration: ClinicalTrials.gov Identifier: NCT02357459.

\section{PLAIN LANGUAGE SUMMARY}

Osteoarthritis is a chronic condition that greatly impacts patients. Pain is the most common symptom of osteoarthritis. Clinical trials evaluating the effects of new drugs to treat osteoarthritis pain frequently use scales to rate overall pain following treatment. Patients may rate their pain using a number that best describes their pain, with the lowest number typically meaning "no pain," and the highest number typically meaning "pain as bad as you can imagine." Other rating scales may be used to rate pain in situations commonly associated with osteoarthritis.

Results from a large clinical trial demonstrated that injection of an extended-release steroid significantly reduced pain compared with a conventional steroid injection on only one of the two pain-reporting scales used in the trial. A closer look found that some patients reported their pain differently on the two rating scales at the start of the trial, with some reporting moderate-to-severe pain using one questionnaire and mild pain using the other. Here, we focused on those patients who reported having moderate-to-severe osteoarthritis knee pain on both pain scales at the start and found that the pain relief benefit associated with the extended-release steroid injection was greatly improved compared with the conventional steroid injection with both measures. Patients receiving the extended-release steroid injection also decreased their use of rescue medication for pain relief.

Keywords: Corticosteroid; Intra-articular; Knee osteoarthritis; Pain; Triamcinolone acetonide extended-release 


\section{Key Summary Points}

\section{Why carry out this study?}

Average daily pain (ADP) using a numeric rating scale (NRS) and Western Ontario and McMaster Universities Osteoarthritis Index (WOMAC-A subscale) are often used to assess osteoarthritis (OA) knee pain.

In a previous phase 3 study of patients with OA of the knee and well-balanced entry demographics, differing efficacy results were observed with the two different pain-reporting instruments when triamcinolone acetonide extendedrelease (TA-ER) was compared with conventional TA crystalline suspension (TAcs).

As trial enrollment criteria may have contributed to that discrepancy, we conducted a post hoc analysis to assess treatment effects in those patients who reported moderate-to-severe OA pain at baseline on both ADP and WOMAC-A (pain) scales (concordant pain reporters).

\section{What was learned from the study?}

In concordant pain reporters, TA-ER provided statistically significant and clinically meaningful pain relief for at least 12 weeks compared with conventional TAcs, suggesting that patients with knee OA pain who rated their pain consistently across two reporting instruments were better able to discern treatment effect.

Results of this post hoc analysis have implications for study design and patient recruitment of future trials evaluating the efficacy of intra-articular interventions for OA knee pain.

\section{INTRODUCTION}

Osteoarthritis (OA) of the knee is a serious and prevalent chronic disease with pain as its primary symptom [1, 2], and analgesic responses are the key endpoints in OA clinical trials [3-5]. The general, single-item, 11-point numeric rating scale (NRS) instrument, which can be used to report average daily pain (ADP) [6, 7], is applicable to a variety of disorders, and as a simple, unidimensional scale, it provides a fast and reliable method for measuring pain that is particularly useful for determining onset of effect $[7,8]$. The disease-specific, five-item Western Ontario and McMaster Universities Osteoarthritis Index pain subscale (WOMAC-A) has also been frequently used in OA clinical trials, as part of a tool that assesses broader OA symptoms of stiffness and functioning (WOMAC-B and WOMAC-C, respectively) [9].

Conventional intra-articular corticosteroid (IACS) injections, such as triamcinolone acetonide crystalline suspension (TAcs), have been shown to reduce pain in patients with knee OA; however, questions remain about the duration and consistency of analgesic treatment effect given the paucity of high-quality prospective clinical trials [10, 11]. A microsphere-based extended-release formulation of triamcinolone acetonide (TA-ER) has consistently shown $\geq 50 \%$ reduction in pain from baseline as measured by ADP and WOMAC-A across several randomized controlled clinical trials in patients with knee OA [12-15], with durations of effect lasting up to 16 weeks when compared to saline placebo $[14,15]$. However, in the pivotal phase 3 trial, when TA-ER was compared with conventional TAcs, differing efficacy results were observed with ADP and WOMAC-A [14]. At week 12, a single intra-articular (IA) injection of TA-ER did not demonstrate a significant difference in ADP intensity as assessed by the NRS tool, but TA-ER significantly $(p<0.05$ ) reduced pain compared with TAcs as assessed via the WOMAC-A subscale at the same landmark week 12 time point [14].

We hypothesized that trial enrollment criteria may have contributed to the inconsistent results observed across pain reporting 
instruments. In this trial, ADP scores $\geq 5$ to $\leq 9$ reflecting moderate-to-severe pain were required for inclusion in the study; however, no qualifying WOMAC-A score was required for randomization. Inclusion of these patients who reported their pain inconsistently across the two instruments may have masked the true treatment effects and complicated interpretation of the phase 3 results. Therefore, we conducted a post hoc analysis focused on assessing treatment effects in those patients who reported moderate-to-severe knee OA pain according to both ADP and WOMAC-A scales (concordant pain reporters), with a secondary focus on those patients from the analysis who reported mild pain at baseline based on the WOMAC-A scale (discordant pain reporters).

\section{METHODS}

\section{Ethics Compliance}

All procedures were conducted in accordance with the ethical standards of the responsible committee on human experimentation (institutional and national) and with the Helsinki Declaration of 1964, as revised in 2013. The study protocol was approved by governing ethics bodies at the participating sites, and patients provided written informed consent before participating in any study-related procedures.

\section{Study Design}

This was a post hoc analysis of a phase 3 multicenter, randomized, double-blind study (ClinicalTrials.gov Identifier NCT02357459). Full details on patient eligibility, study design, and interventions have been reported elsewhere [14]. Briefly, men and women aged $\geq 40$ years with body mass index $\leq 40 \mathrm{~kg} / \mathrm{m}^{2}$ with symptomatic knee OA and balanced demographics were randomly assigned to receive a single IA injection of TA-ER $32 \mathrm{mg}(n=161)$, TAcs $40 \mathrm{mg}$ $(n=161)$, or saline-placebo $(n=162)$. Patients had symptomatic knee OA defined by American College of Rheumatology criteria for $\geq 6$ months [16], Kellgren-Lawrence grade 2/3 OA based on screening index-knee radiography [17], and an average daily baseline ADP score $\geq 5$ to $\leq 9$ for $\geq 5$ of the 7 days prior to study enrollment $[6,7]$. Although captured at baseline, no qualifying WOMAC-A (pain) score was required for enrollment. All patients enrolled in the original study underwent the same protocols regardless of baseline pain reporting.

Patients were defined as concordant pain reporters if they had reported moderate-to-severe pain on both ADP (average daily score $\geq 5$ to $\leq 9$ ) and the WOMAC-A pain subscale (average score $\geq 2)$ at baseline $(n=292 / 484)$. Patients with a baseline ADP score $\geq 5$ to $\leq 9$, but who reported "mild" baseline pain on the WOMAC-A subscale (WOMAC-A < 2) [18] were considered discordant pain reporters $(n=192 /$ 484).

\section{Study Assessments}

ADP intensity was assessed daily on an NRS of 0 (no pain) to 10 (pain as bad as you can imagine) for 7 days prior to study enrollment. Patients called into an interactive voice-response system every day (4:00 p.m. to 12:00 a.m. locally) from screening to week 24 to report their ADP scores. The weekly mean of the mean daily ADP intensity scores was calculated from baseline through week 24. WOMAC-A (pain) scores were assessed using Likert 3.1, five-point subscales ranging from 0 (no symptoms) to 4 (extreme) [9], and measured at baseline and weeks 4, 8, 12, 16,20 , and 24 . If patients were included in either the concordant or discordant group, their ADP and WOMAC-A scores were included regardless of responsiveness to the treatment.

Rescue analgesic medication usage was monitored throughout the study via a daily diary reporting system, and pill counts were confirmed at each clinical visit. The mean number of rescue medication tablets used per week was computed for each patient by summing the number of tablets used in each weekly interval and dividing by the number of days of non-missing responses in the weekly interval. 
Treatment-emergent adverse events (TEAEs)_defined as any adverse event (AE) with onset after the administration of study treatment or any AE that was present at baseline but worsened in intensity through the end of the study-were analyzed.

\section{Statistical Analysis}

The effects of IA injection treatment were measured by least-squares means (LSM) at each week through week 24 for TA-ER vs. saline-placebo and TAcs and evaluated using a longitudinal mixed-effects model for repeated measures (MMRM) with fixed effects for study time point, study site, and baseline pain score. Area-under-the-effect (AUE) curves of change in weekly mean ADP intensity scores from weeks 1 to $12\left(\right.$ AUE $\left._{\text {weeks1-12 }}\right)$ and weeks 1 to 24

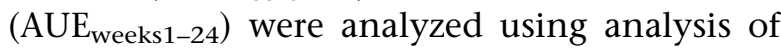
covariance with study site as a covariate.

A direct comparison of the ADP and WOMAC-A pain reporting instruments was carried out based on data from the entire phase 3 population. WOMAC-A scores at baseline were standardized to NRS-approximate equivalents on a 0-10 scale by multiplying WOMAC-A average scores by 2.5. Agreement between the adjusted WOMAC-A and ADP scores was assessed by calculation of Pearson correlation coefficients $(r)$. Effect sizes based on $r$ statistics are generally considered to be small, medium, or large based on cutoffs of $0.10,0.30$, and 0.50 , respectively [19].

\section{RESULTS}

\section{Patient Disposition and Baseline Characteristics}

This post hoc analysis identified 292 patients (TA-ER, $n=95$; saline-placebo, $n=97$; TAcs, $n=100$ ) from the phase 3 full analysis set (FAS; $n=484$ ) who reported moderate-to-severe knee OA pain at baseline on both ADP and WOMACA (pain) scales (ADP $\geq 5$ to $\leq 9$ and WOMAC$\mathrm{A} \geq 2$; concordant pain reporters); 192 patients (39.7\%) who reported moderate-to-severe knee
OA on the ADP scale but mild knee OA pain on the WOMAC-A scale (discordant pain reporters) were also assessed. Patient demographics and baseline characteristics are shown in Table 1. Patients ranged from 40 to 83 years of age (mean, 61.1 years), the majority were female $(62.3 \%)$, and had a mean body mass index of approximately $30 \mathrm{~kg} / \mathrm{m}^{2}$. Figure 1 shows the correlation of ADP and WOMAC-A scores at baseline for the entire phase 3 study population (FAS; $n=484$ ). After normalization to a common scale, ADP intensity scores using the NRS were approximately 23\% higher than WOMACA scores at baseline (Pearson correlation coefficient $[r]=0.402)$.

\section{Treatment Outcomes}

For concordant pain reporters, change from baseline in ADP score was significantly $(p<0.05)$ improved with TA-ER compared with TAcs each week from weeks 5 to 19 and compared with saline-placebo each week from weeks 1 to 20 (Fig. 2). Analysis of the frequency distribution of ADP intensity scores demonstrated that $\sim 28 \%$ of patients treated with TA-ER reported that they had no pain at week 12 , compared with only $\sim 8 \%$ of patients who received TAcs (Fig. 3). Lack of pain was maintained at week 16 in $\sim 20 \%$ of patients treated with TA-ER (compared with 9\% of patients who received TAcs) (data not shown).

For concordant pain reporters, LSM differences $(95 \% \mathrm{CI})$ in change from baseline in ADP score at week 12 were greater (TA-ER vs. TAcs: $-0.87[-1.54,-0.20], p=0.0105$; TA-ER vs. saline-placebo: $-1.75 \quad[-2.44, \quad-1.06]$, $p<0.0001)$ than for discordant pain reporters (TA-ER vs. TAcs: $0.45[-0.28,1.17], p=0.2285$; TA-ER vs. saline-placebo: -0.01 [-0.73, 0.70]; $p=0.9707$ ) (Table 2).

The AUE for the ADP intensity curves reflects the totality of the treatment effect. For concordant pain reporters, AUE $_{\text {weeks1-12 }}$ and AUE $_{\text {weeks1-24 }}$ treatment effects were statistically significant for TA-ER compared with TAcs (LSM differences [95\% CI]: $-47.7[-94.4,-1.0]$ and $-98.4[-194.5,-2.3]$, respectively; $p<0.05$ for both) and TA-ER compared with saline-placebo 
Table 1 Demographic and baseline characteristics of concordant pain reporters

\begin{tabular}{|c|c|c|c|}
\hline & $\begin{array}{l}\text { TA-ER } \\
32 \mathrm{mg} \\
n=95\end{array}$ & $\begin{array}{l}\text { Saline-placebo } \\
n=97\end{array}$ & $\begin{array}{l}\text { TAcs } \\
40 \mathrm{mg} \\
n=100\end{array}$ \\
\hline \multicolumn{4}{|l|}{ Sex, $n(\%)$} \\
\hline Male & $33(34.7)$ & $37(38.1)$ & $40(40.0)$ \\
\hline Female & $62(65.3)$ & $60(61.9)$ & $60(60.0)$ \\
\hline Age, years, mean (SD) & $61.1(9.2)$ & $61.3(8.9)$ & $61.0(10.1)$ \\
\hline \multicolumn{4}{|l|}{ Race, $n(\%)$} \\
\hline American Indian or Alaska Native & 0 & 0 & 0 \\
\hline Asian & $9(9.5)$ & $9(9.3)$ & $10(10.0)$ \\
\hline Black or African American & $9(9.5)$ & $3(3.1)$ & $9(9.0)$ \\
\hline Native Hawaiian or other Pacific Islander & $3(3.2)$ & $1(1.0)$ & $2(2.0)$ \\
\hline White & $74(77.9)$ & $84(86.6)$ & $79(79.0)$ \\
\hline Other & 0 & 0 & 0 \\
\hline BMI $\left(\mathrm{kg} / \mathrm{m}^{2}\right)$, mean $(\mathrm{SD})$ & $30.3(5.0)$ & $30.4(4.8)$ & $30.9(4.7)$ \\
\hline Years since primary diagnosis, mean (SD) & $8.3(7.4)$ & $6.3(5.8)$ & $7.6(7.0)$ \\
\hline \multicolumn{4}{|l|}{ Kellgren-Lawrence grade, $n(\%)$} \\
\hline 2 & $49(51.6)$ & $44(45.4)$ & $43(43.0)$ \\
\hline 3 & $46(48.4)$ & $53(54.6)$ & $56(56.0)$ \\
\hline 4 & 0 & 0 & $1(1.0)$ \\
\hline \multicolumn{4}{|l|}{ Unilateral/bilateral knee OA, $n(\%)$} \\
\hline Unilateral & $33(34.7)$ & $37(38.1)$ & $42(42.0)$ \\
\hline Bilateral & $62(65.3)$ & $60(61.9)$ & $58(58.0)$ \\
\hline Weekly ADP intensity score at baseline, mean (SD) & $6.42(0.94)$ & $6.54(1.01)$ & $6.49(0.95)$ \\
\hline WOMAC-A (pain) score, mean (SD) & $2.37(0.34)$ & $2.36(0.35)$ & $2.37(0.32)$ \\
\hline
\end{tabular}

$A D P$ average daily pain, $B M I$ body mass index, $O A$ osteoarthritis, $S D$ standard deviation, $T A c s$ triamcinolone acetonide crystalline suspension, $T A-E R$ triamcinolone acetonide extended-release, WOMAC Western Ontario and McMaster Universities Osteoarthritis Index

$(-136.1[-184.2,-88.0]$ and $-212.1[-311.1$, -113.1], respectively; $p<0.0001$ for both) (Table 2). For comparison, among discordant pain reporters, LSM differences (95\% CI) in AUE $_{\text {weeks1-12 }}$ and AUE $_{\text {weeks1-24 }}$ for TA-ER compared with TAcs were $12.3(-41.6,66.3$; $p=0.6518)$ and $77.1(-26.1,180.2 ; p=0.1419)$, respectively, and LSM differences for TA-ER compared with saline-placebo were -58.2 $(-111.2,-5.3 ; p<0.05)$ and $-37.7(-139.0$, 63.6; $p=0.4635)$, respectively.

In concordant pain reporters, TA-ER also demonstrated statistically significant $(p<0.05)$ improvements over both TAcs and saline-placebo in WOMAC-A (pain) scores (weeks 4, 8, and 12 vs. TAcs; weeks 4, 8, 12, and 16 vs. 


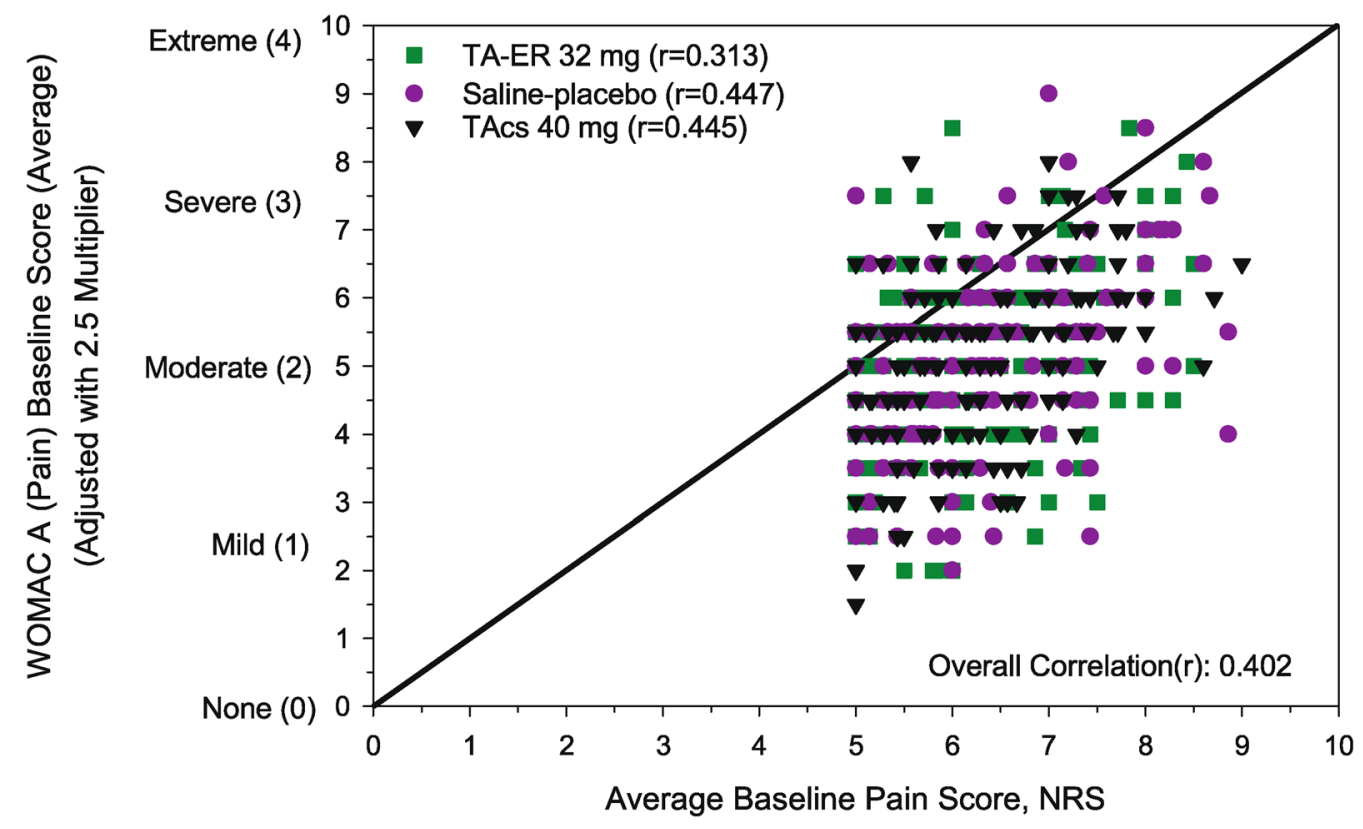

Fig. 1 Correlation between ADP and normalized WOMAC-A-derived baseline pain assessments in the phase 3 full analysis set. NRS numeric rating scale, TAcs triamcinolone acetonide crystalline suspension, $T A-E R$ triamcinolone acetonide extended-release, WOMAC Western Ontario and McMaster Universities Osteoarthritis Index. Data from the phase 3 FAS population: TA-ER $(n=161)$, saline-placebo $(n=162)$, TAcs $(n=161)[14]$

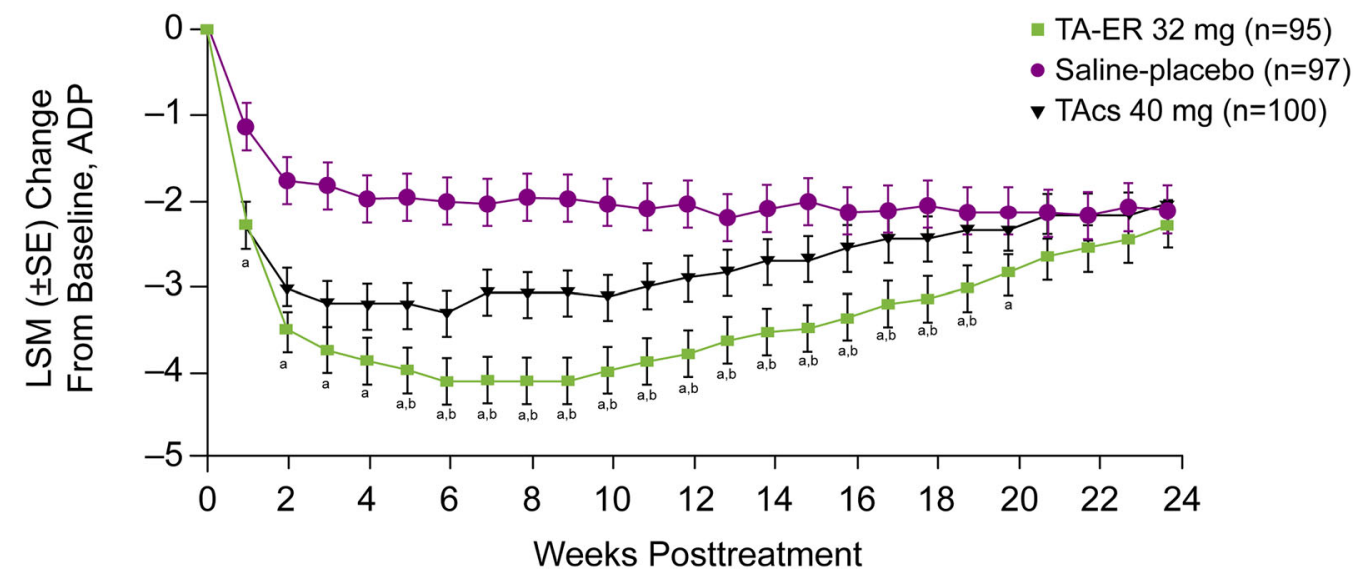

Fig. 2 Mean changes from baseline in ADP scores over time among concordant pain reporters. ${ }^{a} p<0.05$ vs. saline-placebo; ${ }^{\mathrm{b}} p<0.05$ vs. TAcs. ADP average daily

saline-placebo) (Fig. 4). For concordant pain reporters, TA-ER reduced pain (WOMAC-A) from baseline by as much as 59\% (week 4); the largest reduction in pain resulting from TAcs treatment was $46 \%$ (week 4 ). In contrast, among discordant pain reporters, mean changes from pain, LSM least-squares mean, TAcs triamcinolone acetonide crystalline suspension, $T A-E R$ triamcinolone acetonide extended-release

baseline in WOMAC-A scores were not statistically significant vs. TAcs at any time point ( $p>0.1$ for weeks $4,8,12,16,20$, and 24); changes were statistically significant vs. salineplacebo at week 4 only $(p=0.0011)$. 


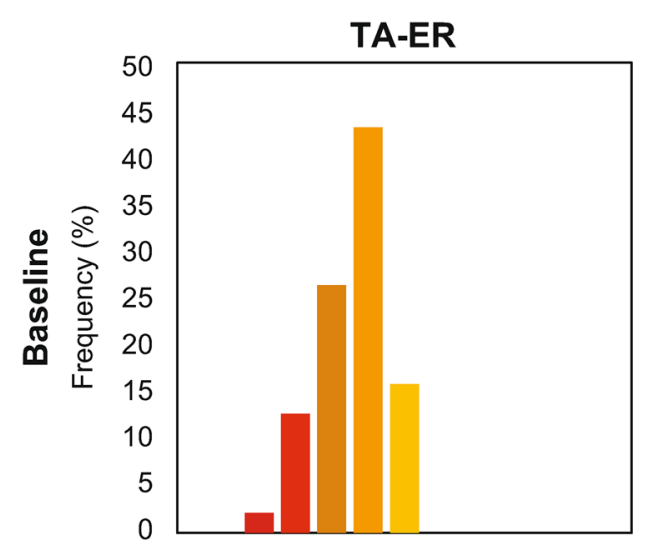

TAcs
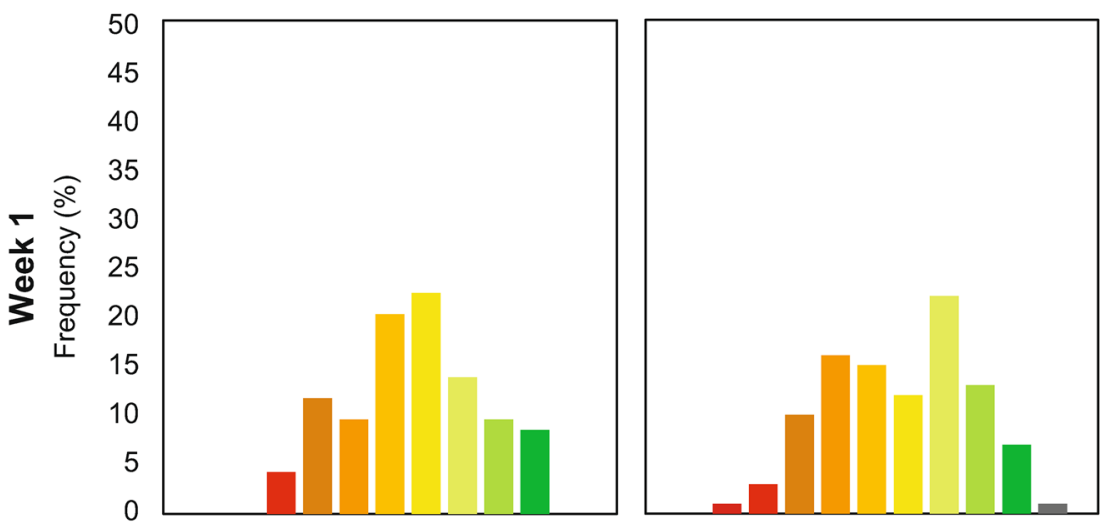

10 NRS 10 (Severe Pain)

- 9 NRS 9 (Severe Pain)

- 8 NRS 8 (Severe Pain)

7 NRS 7 (Moderate Pain)

6 NRS 6 (Moderate Pain)

5 NRS 5 (Moderate Pain)

4 NRS 4 (Mild Pain)

3 NRS 3 (Mild Pain)

- 2 NRS 2 (Mild Pain)

- 1 NRS 1 (Mild Pain)

- 0 NRS 0 (No Pain)
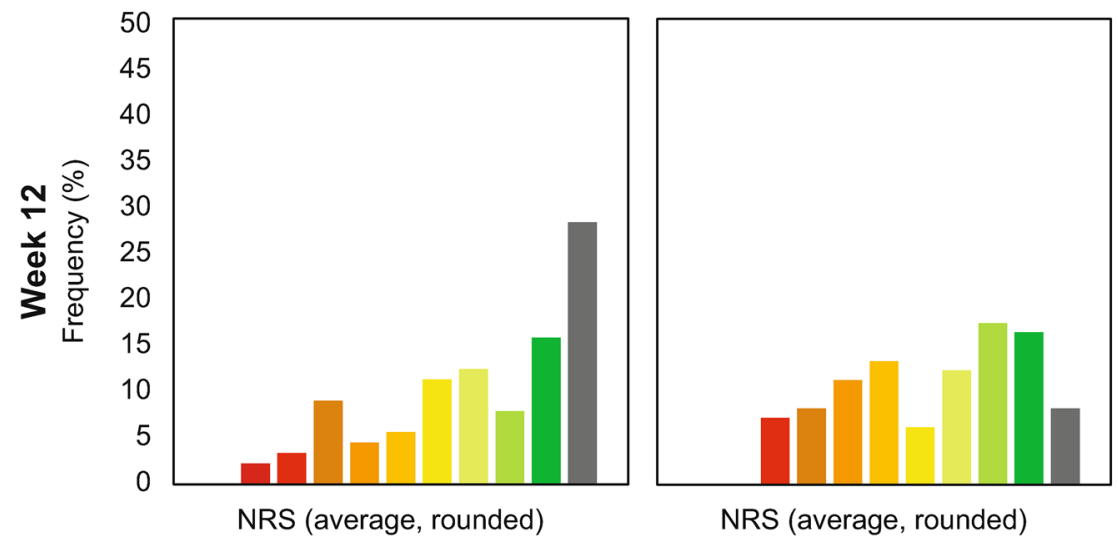

Fig. 3 Frequency distribution of ADP scores at baseline, week 1, and week 12 among concordant pain reporters. ADP scores rated on a $0-10$ NRS, with 0 indicating "no pain” and 10 indicating "pain as bad as you can imagine."

$A D P$ average daily pain, $N R S$ numeric rating scale, $T A c s$ triamcinolone acetonide crystalline suspension, $T A-E R$ triamcinolone acetonide extended-release

Concordant pain reporters treated with TAER used significantly fewer rescue medication tablets each week from weeks 2 to 20 compared with TAcs and each week from weeks 1 to 24 compared with saline-placebo (Fig. 5). Among

discordant pain reporters, no significant differences were noted between TA-ER and TAcs or between TA-ER and saline-placebo at any time point ( $p>0.05$ vs. both TAcs and saline-placebo at all time points). 
Table 2 Differences in ADP scores at week 12 and area-under-the-effect curve

\begin{tabular}{|c|c|c|c|c|c|c|}
\hline & \multicolumn{6}{|c|}{ LSM difference in ADP (95\% CI); $p$ value } \\
\hline & \multicolumn{2}{|c|}{$\begin{array}{l}\text { Concordant pain reporters } \\
\text { TA-ER }(n=95) \\
\text { Saline-placebo }(n=97) \\
\text { TAcs }(n=100)\end{array}$} & \multicolumn{2}{|c|}{$\begin{array}{l}\text { Discordant pain reporters } \\
\text { TA-ER }(n=66) \\
\text { Saline-placebo }(n=65) \\
\text { TAcs }(n=61)\end{array}$} & \multicolumn{2}{|c|}{$\begin{array}{l}\text { Phase } 3 \text { FAS }^{\mathrm{a}} \\
\text { TA-ER }(n=161) \\
\text { Saline-placebo }(n=162) \\
\text { TAcs }(n=161)\end{array}$} \\
\hline & $\begin{array}{l}\text { TA-ER vs. } \\
\text { saline-placebo }\end{array}$ & $\begin{array}{l}\text { TA-ER vs. } \\
\text { TAcs }\end{array}$ & $\begin{array}{l}\text { TA-ER vs. } \\
\text { saline-placebo }\end{array}$ & $\begin{array}{l}\text { TA-ER vs. } \\
\text { TAcs }\end{array}$ & $\begin{array}{l}\text { TA-ER vs. } \\
\text { saline-placebo }\end{array}$ & $\begin{array}{l}\text { TA-ER vs. } \\
\text { TAcs }\end{array}$ \\
\hline \multirow{3}{*}{$\begin{array}{l}\text { LSM } \\
\text { difference } \\
\text { at week } 12\end{array}$} & -1.75 & -0.87 & -0.01 & 0.45 & -0.98 & -0.26 \\
\hline & $(-2.44,-1.06)$ & $(-1.54,-0.20)$ & $(-0.73,0.70)$ & $(-0.28,1.17)$ & $(-1.47,-0.49)$ & $(-0.74,0.23)$ \\
\hline & $<0.0001$ & 0.0105 & 0.9707 & 0.2285 & $<0.0001$ & 0.2964 \\
\hline \multirow[t]{3}{*}{ AUE $_{\text {weeks1-12 }}$} & -136.1 & -47.7 & -58.2 & 12.3 & -102.0 & -15.3 \\
\hline & $(-184.2,-88.0)$ & $(-94.4,-1.0)$ & $(-111.2,-5.3)$ & $(-41.6,66.3)$ & $(-136.8,-67.3)$ & $(-49.8,19.2)$ \\
\hline & $<0.0001$ & 0.0451 & 0.0314 & 0.6518 & $<0.0001$ & 0.3827 \\
\hline \multirow[t]{3}{*}{$\mathrm{AUE}_{\mathrm{weeks1-24}}$} & -212.1 & -98.4 & -37.7 & 77.1 & -135.5 & -13.2 \\
\hline & $(-311.1,-113.1)$ & $(-194.5,-2.3)$ & $(-139.0,63.6)$ & $(-26.1,180.2)$ & $(-205.9,-65.2)$ & $(-83.0,56.7)$ \\
\hline & $<0.0001$ & 0.0447 & 0.4635 & 0.1419 & 0.0002 & 0.7111 \\
\hline
\end{tabular}

${ }^{\mathrm{a}}$ Data from the phase 3 FAS population [14]

$A D P$ average daily pain, $A U E$ area-under-the-effect curve, $C I$ confidence interval, $F A S$ full analysis set, $L S M$ least-squares mean, TAcs triamcinolone acetonide crystalline suspension, $T A$-ER triamcinolone acetonide extended-release

\section{Safety}

Safety profiles were similar among treatment groups and consistent with those of the overall phase 3 safety population (Table 3) [14]. The incidence of TEAEs was comparable between treatment groups, with most TEAEs being grade 1 or 2, non-serious, and unrelated to study drug. The one exception was index-knee TEAEs, which largely comprises reports of mild-moderate arthralgia, wherein $19 \%$ of those receiving TA-ER showed $\geq 1$ index-knee TEAE, compared with $9 \%$ and $10 \%$ of those receiving saline-placebo and TAcs, respectively (Table 3 ). Indexknee arthralgia is notable in the mean timing of onset in the three treatment groups (21.5 days for saline-placebo, 57 days for TAcs, and 78 days for TA-ER), suggesting these reports may represent waning of pain relief.

\section{DISCUSSION}

In a large phase 3 study of TA-ER, although the study population was limited, as intended, to patients with moderate-to-severe $\mathrm{OA}$ pain at baseline (based on ADP score $\geq 5$ to $\leq 9$ ), nearly $40 \%$ of the patients had reported their pain as "mild" on the WOMAC-A scale $(<2)$. In patients who reported moderate-to-severe baseline knee OA pain as assessed using both ADP $(\geq 5$ to $\leq 9)$ and WOMAC-A $(\geq 2)$ instruments, a single IA TA-ER injection provided statistically significant $(p<0.05)$ improvements in patientreported OA outcomes compared with TAcs and saline-placebo. These included reductions in pain and rescue medication usage. TA-ER treatment effects generally persisted for 16 weeks or longer; an ADP-based AUE analysis showed significant differences between TA-ER and both 


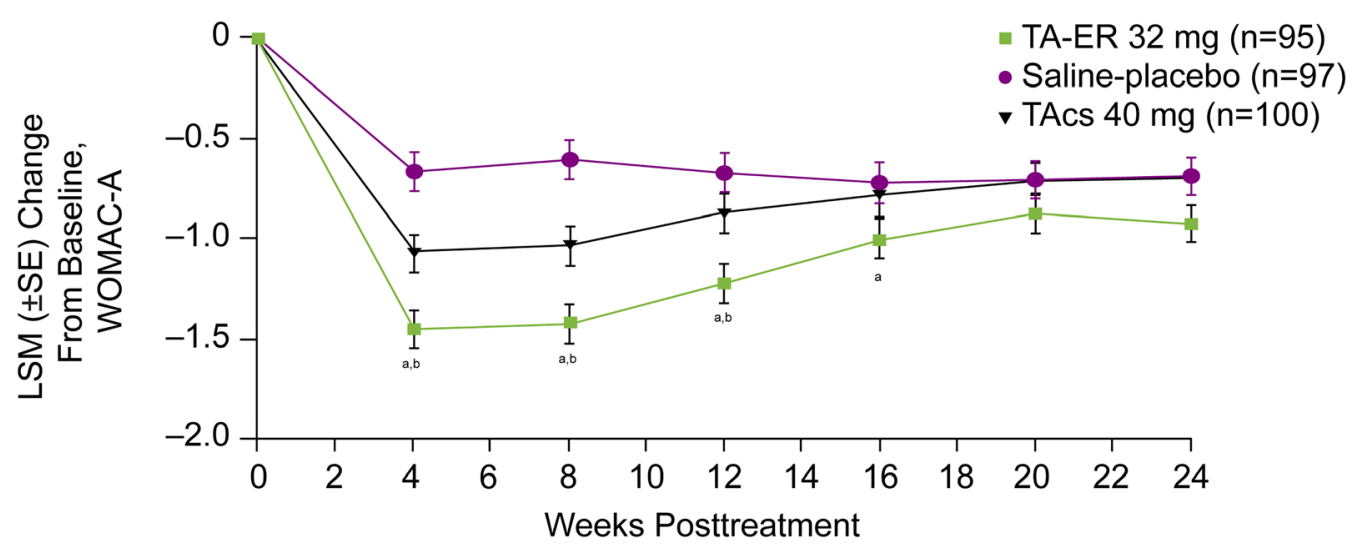

Fig. 4 Mean change from baseline in WOMAC-A (pain) score among concordant pain reporters. ${ }^{a} p<0.05$ vs. saline-placebo; ${ }^{\mathrm{b}} p<0.05$ vs. TAcs. $L S M$ least-squares mean, TAcs triamcinolone acetonide crystalline suspension,
TA-ER triamcinolone acetonide extended-release, WOMAC Western Ontario and McMaster Universities Osteoarthritis Index

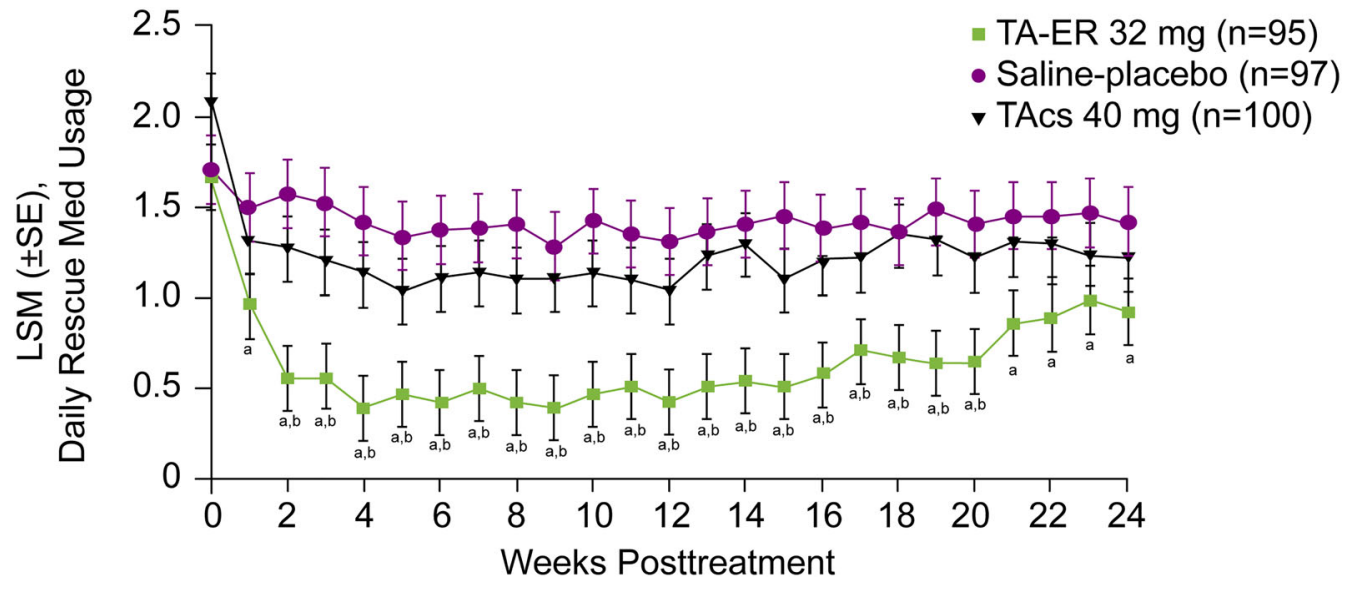

Fig. 5 Mean rescue medication usage among concordant pain reporters. ${ }^{\mathrm{a}} p<0.05$ vs. saline-placebo; ${ }^{\mathrm{b}} p<0.05$ vs. TAcs. LSM least-squares mean, TAcs triamcinolone

comparators through week 24 , confirming the totality of the TA-ER treatment effect over time. The LSM difference at 12 weeks and the AUE for ADP results were enhanced in concordant pain reporters compared with those observed in the phase 3 FAS (Table 2) [14]. These results, demonstrating the persistence of the TA-ER effect, are consistent with the mechanism of action of an extended-release IACS [20, 21].

The magnitude of pain relief associated with TA-ER was substantial. TA-ER reduced pain (based on ADP intensity) from baseline by $\geq 50 \%$ from weeks 2 to 16 (inclusive), whereas TAcs reduced pain by $\geq 50 \%$ at week 6 only. acetonide crystalline suspension, TA-ER triamcinolone acetonide extended-release

There was a clear shift in the distribution of ADP intensity scores toward mild-to-no-pain from baseline to week 12 with both TA-ER and TAcs treatment; however, more than three times as many patients receiving TA-ER reported no knee pain at week 12 compared with TAcs. The magnitude of this TA-ER treatment effect was sustained through week 16 , with approximately $20 \%$ of patients in the TA-ER group still reporting no pain.

The magnitude and duration of the analgesic effects of TA-ER observed in this post hoc analysis have not been attained, to our knowledge, in studies evaluating conventional IACS 
Table 3 Summary of adverse events among concordant pain reporters

\begin{tabular}{|c|c|c|c|}
\hline & $\begin{array}{l}\text { TA-ER } \\
32 \mathrm{mg} \\
n=95\end{array}$ & $\begin{array}{l}\text { Saline-placebo } \\
n=97\end{array}$ & $\begin{array}{l}\text { TAcs } \\
40 \mathrm{mg} \\
n=100\end{array}$ \\
\hline$\geq 1$ TEAE, $n(\%)$ & $50(52.6)$ & $48(49.5)$ & $56(56.0)$ \\
\hline Grade 1 & $19(20.0)$ & $20(20.6)$ & $20(20.0)$ \\
\hline Grade 2 & $25(26.3)$ & $26(26.8)$ & $33(33.0)$ \\
\hline Grade 3 & $5(5.3)$ & $2(2.1)$ & $3(3.0)$ \\
\hline Grade 4 & $1(1.1)$ & 0 & 0 \\
\hline$\geq 1$ serious TEAE & $5(5.3)$ & $2(2.1)$ & $3(3.0)$ \\
\hline$\geq 1$ TEAE leading to study discontinuation & 0 & 0 & $1(1.0)$ \\
\hline \multicolumn{4}{|l|}{ TEAE by maximum relationship } \\
\hline Not related ${ }^{\mathrm{a}}$ & $45(47.4)$ & $45(46.4)$ & $52(52.0)$ \\
\hline Related $^{\mathrm{b}}$ & $5(5.3)$ & $3(3.1)$ & $4(4.0)$ \\
\hline$\geq 1$ index-knee TEAE & $18(18.9)$ & $9(9.3)$ & $10(10.0)$ \\
\hline Index-knee arthralgia & $11(11.6)$ & $6(6.2)$ & $4(4.0)$ \\
\hline$\geq 1$ index-knee TEAE leading to study discontinuation & 0 & 0 & $1(1.0)$ \\
\hline
\end{tabular}

${ }^{a}$ Includes "not related" and "unlikely" related

bIncludes "possibly," "probably," or "definitely" related

$A D P$ average daily pain, $T A c s$ triamcinolone acetonide crystalline suspension, TA-ER triamcinolone acetonide extendedrelease, TEAE treatment-emergent adverse event

injections. These drugs are often associated with only moderate treatment effects and typically offer pain relief that is $<6$ weeks in duration [10]. Results of a recent meta-analysis suggest that although smaller studies tend to demonstrate a moderate pain-relief benefit of conventional IACS injections, such benefits were relatively diminished among the few moderateto-large trials ( $\geq 50$ patients per trial group) that have been conducted so far $[10,11]$. In addition, in this analysis the treatment groups were balanced in numbers of patients with unilateral or bilateral knee OA, meaning that treatment effects observed are not subject to differences associated with patients with unilateral disease being better able to self-assess changes in index knee pain than patients with bilateral disease [22].

The magnitude and duration of pain relief observed in this post hoc analysis were enhanced compared with those observed for the phase 3 FAS [14], suggesting that patients with concordant reporting of moderate-to-severe OA pain at baseline were better able to discern treatment effect. Instrument characteristics may have played a role in the variability of the patient's assessment of their pain at baseline. The ADP tool is not disease-specific, and data is captured using a single-item measure (11-point NRS scale) $[6,7]$. This is in contrast to pain outcomes captured using the WOMAC-A tool, which assesses joint pain related to walking, using stairs, being in bed, sitting or lying down, and standing (each item scored individually) [9]. Results of this study have implications for the design of future trials to evaluate the efficacy of analgesics. In particular, the results highlight important considerations for patient recruitment and enrollment, such as the need to confirm the presence of moderate-to-severe pain by using more than one assessment tool. Further, results of this study emphasize 
potential value of keeping both patients and trial sites blinded to study enrollment criteria as it relates to pain scores in order to minimize potential for inflated baseline pain scores.

The current analysis is limited in its post hoc, retrospective nature, which makes the applicability and generalizability of the findings unclear. Prospective studies are needed to confirm the results obtained.

\section{CONCLUSIONS}

TA-ER provided statistically significant and clinically meaningful pain relief for at least 12 weeks compared with conventional TAcs in patients who reported moderate-to-severe knee OA pain at baseline concordantly on two different scales (ADP for consistency of pain response and WOMAC-A for pain measurement specifically in OA). Treatment effects were enhanced compared with those observed for the phase 3 FAS, suggesting that patients with concordant reporting of pain across two instruments were better able to discern treatment effect. In these patients, TA-ER reduced pain from baseline by $\geq 50 \%$ extending to week 16 , with approximately $20 \%$ of patients reporting no pain at week 16 . The magnitude and duration of pain relief observed with TA-ER in this study highlights the potential substantial benefits over conventional TAcs. Results of this analysis also have implications for study design and patient recruitment of future trials evaluating efficacy of analgesic interventions for OA knee pain. Trial and instrument characteristics, including pain-based eligibility criteria, scale differences, and measurement variability, must be considered when selecting instruments and end points in clinical trials comparing the magnitude of analgesic benefit observed between active therapies.

\section{ACKNOWLEDGEMENTS}

The authors thank the participants in the study. The authors thank James R. Johnson, PhD, (formerly employed by Summit Analytical, Denver, CO) for biostatistical support. PGC is supported in part by the National Institute for Health Research (NIHR) Leeds Biomedical Research Centre. The views expressed are those of the author(s) and not necessarily those of the NHS, the NIHR, or the Department of Health.

Funding. This analysis and all processing charges, including the journal's Rapid Service Fee, were funded by Flexion Therapeutics, Inc., Burlington, MA, USA. All authors had full access to all the data in this analysis and take complete responsibility for the integrity of the data and accuracy of the data analysis.

Medical Writing and Editorial Assistance. Professional medical writing and editorial assistance in the preparation of this article was provided by Anna Battershill and Thomas C. Rotolo, PhD (ApotheCom, Yardley, PA, USA). Support for this assistance was funded by Flexion Therapeutics, Inc. (Burlington, MA, USA).

Authorship. All named authors meet the International Committee of Medical Journal Editors (ICMJE) criteria for authorship for this article, take responsibility for the integrity of the work as a whole, and have given their approval for this version to be published.

Authors' Contributions. All authors contributed to the study conception and design. Data analysis was performed by Amy Cinar. All authors reviewed and provided feedback on previous versions of the manuscript and provided approval on the final version.

Prior Presentation. These data in part have been presented previously at the American Pain Society Annual Meeting, April 3-6, 2019 (Milwaukee, WI); American College of Rheumatology Annual Meeting, November 8-13, 2019 (Atlanta, GA); PAINWeek, September 3-7, 2019 (Las Vegas, NV); American Medical Society for Sports Medicine, April 24-29, 2020 (Atlanta, GA); Osteoarthritis Research Society International, April 30-May 3, 2020 (Vienna, Austria), and Western Orthopaedic Association, August 5-8, 2020 (Maui, HI). 
Disclosures. Edgar Ross has no conflicts of interest to disclose. Nathaniel P. Katz received consulting fees from Flexion Therapeutics, Inc., paid to WCG Analgesic Solutions, where he serves as Chief Science Officer. Philip G. Conaghan has received compensation for consultancies from AbbVie, Amgen, Eli Lilly, Flexion Therapeutics, Inc., Merck Serono, Novartis, Pfizer, and Stryker. Alan Kivitz is a shareholder of Amgen, Gilead, GlaxoSmithKline, Novartis, Pfizer, and Sanofi. He has received consulting fees from AbbVie, Boehringer Ingelheim, Flexion Therapeutics, Inc., Gilead, Janssen, Pfizer, Regeneron, Sanofi, and SUN Pharma Advanced Research. He has also served as a paid presenter or speaker for AbbVie, Celgene, Flexion Therapeutics, Inc., Genzyme, GlaxoSmithKline, Lilly, Merck, Novartis, Pfizer, Sanofi, and UCB. Dennis C. Turk has received research support from the National Institutes of Health and the US Food and Drug Administration. He has also received compensation for consultancies from Flexion Therapeutics, Inc., Eli Lilly, GSK/ Novartis, and Pfizer (all $<\$ 10,000)$. He also serves as editor-in-chief of the Clinical Journal of Pain. Andrew I. Spitzer has received research support from DePuy and Flexion Therapeutics, Inc., and consulting fees from AO Recon, DePuy, and Flexion Therapeutics, Inc., Deryk Jones has received research support from Genzyme and Sanofi-Aventis and consulting fees from Arthrex, CONMED Linvatec, Flexion Therapeutics, Inc., Genzyme, Mitek, Musculoskeletal Transplant Foundation, Tissue Tech, and Zimmer. Ryan K. Lanier received consulting fees from Flexion Therapeutics, Inc., paid to WCG Analgesic Solutions, where he was formerly an employee. Ryan $\mathrm{K}$. Lanier is now an employee of Pinney Associates, Inc., in Bethesda, MD, USA. Amy Cinar is an employee of Flexion Therapeutics, Inc. and owns stock/stock options in Flexion Therapeutics, Inc. Joelle Lufkin and Scott D. Kelley are former employees of Flexion Therapeutics, Inc. and own stock in Flexion Therapeutics, Inc. Joelle Lufkin is now an independent consultant in Beverly, MA, USA. Scott D. Kelley is now affiliated with Brigham and Women's Hospital in Boston, MA, USA.
Compliance with Ethics Guidelines. All procedures were conducted in accordance with the ethical standards of the responsible committee on human experimentation (institutional and national) and with the Helsinki Declaration of 1964, as revised in 2013. The clinical study protocol was approved by governing ethical bodies at the participating sites and patients provided written informed consent before any study-related procedures.

Data Availability. The datasets generated and/or analyzed during the current study are available from the corresponding author on reasonable request.

Open Access. This article is licensed under a Creative Commons Attribution-NonCommercial 4.0 International License, which permits any non-commercial use, sharing, adaptation, distribution and reproduction in any medium or format, as long as you give appropriate credit to the original author(s) and the source, provide a link to the Creative Commons licence, and indicate if changes were made. The images or other third party material in this article are included in the article's Creative Commons licence, unless indicated otherwise in a credit line to the material. If material is not included in the article's Creative Commons licence and your intended use is not permitted by statutory regulation or exceeds the permitted use, you will need to obtain permission directly from the copyright holder. To view a copy of this licence, visit http://creativecommons.org/licenses/by$\mathrm{nc} / 4.0 /$.

\section{REFERENCES}

1. Centers for Disease Control and Prevention. Osteoarthritis Fact Sheet. Last reviewed July 27, 2020. Accessed August 5, 2021. https://www.cdc. gov/arthritis/basics/osteoarthritis.htm.

2. Osteoarthritis Research Society International (OARSI). Osteoarthritis: A Serious Disease. Submitted to the U.S. Food and Drug Administration December 1, 2016. Accessed August 5, 2021. https://www.oarsi.org/sites/default/files/docs/2016/ 
oarsi_white_paper_oa_serious_disease_121416_1. pdf.

3. US Department of Health and Human Services. Guidance for Industry: Analgesic Indications: Developing Drug and Biological Products [Draft Guidance]. Silver Spring: Center for Drug Evaluation and Research (CDER); February 2014.

4. Reginster JY, Reiter-Niesert S, Bruyere O, Berenbaum F, Brandi ML, Branco J, et al. Recommendations for an update of the 2010 European regulatory guideline on clinical investigation of medicinal products used in the treatment of osteoarthritis and reflections about related clinically relevant outcomes: expert consensus statement. Osteoarthr Cartil. 2015;23:2086-93.

5. Smith TO, Hawker GA, Hunter DJ, March LM, Boers $\mathrm{M}$, Shea BJ, et al. The OMERACT-OARSI core domain set for measurement in clinical trials of hip and/or knee osteoarthritis. J Rheumatol. 2019;46:981-9.

6. Cleeland CS, Ryan KM. Pain assessment: global use of the Brief Pain Inventory. Ann Acad Med Singapore. 1994;23:129-38.

7. Dworkin RH, Turk DC, Farrar JT, Haythornthwaite JA, Jensen MP, Katz NP, et al. Core outcome measures for chronic pain clinical trials: IMMPACT recommendations. Pain. 2005;113:9-19.

8. Younger J, McCue R, Mackey S. Pain outcomes: a brief review of instruments and techniques. Curr Pain Headache Rep. 2009;13:39-43.

9. Bellamy N, Buchanan WW, Goldsmith CH, Campbell J, Stitt LW. Validation study of WOMAC: a health status instrument for measuring clinically important patient relevant outcomes to antirheumatic drug therapy in patients with osteoarthritis of the hip or knee. J Rheumatol. 1988;15:1833-40.

10. Juni P, Hari R, Rutjes AW, Fischer R, Silletta MG, Reichenbach $S$, et al. Intra-articular corticosteroid for knee osteoarthritis. Cochrane Database Syst Rev. 2015;10: CD005328.

11. da Costa BR, Hari R, Juni P. Intra-articular corticosteroids for osteoarthritis of the knee. JAMA. 2016;316(24):2671-2.

12. Bodick N, Lufkin J, Willwerth C, Kumar A, Bolognese J, Schoonmaker C, et al. An intra-articular, extended-release formulation of triamcinolone acetonide prolongs and amplifies analgesic effect in patients with osteoarthritis of the knee: a randomized clinical trial. J Bone Joint Surg Am. 2015;97: 877-88.

13. Conaghan PG, Cohen SB, Berenbaum F, Lufkin J, Johnson JR, Bodick N. Phase $2 b$ trial of a novel extended-release microsphere formulation of triamcinolone acetonide for intra-articular injection in knee osteoarthritis. Arthritis Rheumatol. 2018;70:204-11.

14. Conaghan PG, Hunter DJ, Cohen SB, Kraus VB, Berenbaum F, Lieberman JR, et al. Effects of a single intra-articular injection of a microsphere formulation of triamcinolone acetonide on knee osteoarthritis pain: a double-blinded, randomized, placebo-controlled, multinational study. J Bone Joint Surg Am. 2018;100:666-77.

15. Spitzer AI, Richmond JC, Kraus VB, Gomoll A, Jones DG, Huffman KM, et al. Safety and efficacy of repeat administration of triamcinolone acetonide extended-release in osteoarthritis of the knee: a phase $3 \mathrm{~b}$, open-label study. Rheumatol Ther. 2019;6:109-24.

16. Altman R, Asch E, Bloch D, Bole G, Borenstein D, Brandt $K$, et al. Development of criteria for the classification and reporting of osteoarthritis. Classification of osteoarthritis of the knee. Diagnostic and Therapeutic Criteria Committee of the American Rheumatism Association. Arthritis Rheumatol. 1986;29:1039-49.

17. Kellgren JH, Lawrence JS. Radiological assessment of osteo-arthrosis. Ann Rheum Dis. 1957;16: 494-502.

18. Kapstad H, Hanestad BR, Langeland N, Rustoen T, Stavem K. Cutpoints for mild, moderate and severe pain in patients with osteoarthritis of the hip or knee ready for joint replacement surgery. BMC Musculoskelet Disord. 2008;9:55.

19. Cohen J. Statistical power analysis for the behavioral sciences. 2nd ed. Hillsdale: Lawrence Erlbaum Associates; 1988.

20. Kraus VB, Conaghan PG, Aazami HA, Mehra P, Kivitz AJ, Lufkin J, et al. Synovial and systemic pharmacokinetics (PK) of triamcinolone acetonide (TA) following intra-articular (IA) injection of an extended-release microsphere-based formulation (FX006) or standard crystalline suspension in patients with knee osteoarthritis (OA). Osteoarthr Cartil. 2018;26:34-42.

21. Zilretta $^{\mathrm{TM}}$ (triamcinolone acetonide extended-release injectable suspension) [package insert]. Burlington: Flexion Therapeutics, Inc.; January 2020.

22. Langworthy MJ, Conaghan PG, Ruane JJ, Kivitz AJ, Lufkin J, Cinar A, et al. Efficacy of triamcinolone acetonide extended-release in participants with unilateral knee osteoarthritis: a post hoc analysis. Adv Ther. 2019;36:1398-411. 